\title{
D'un humanisme anti-lyrique : « la bêtise de la musique » selon Milan Kundera
}

Bertrand Vibert

\section{(2) OpenEdition}

Journals

Édition électronique

URL : http://journals.openedition.org/recherchestravaux/451

DOI : 10.4000/recherchestravaux.451

ISSN : 1969-6434

Éditeur

UGA Éditions/Université Grenoble Alpes

Édition imprimée

Date de publication : 15 mai 2011

Pagination : $97-116$

ISBN : 978-2-84310-200-4

ISSN : 0151-1874

Référence électronique

Bertrand Vibert, « D'un humanisme anti-lyrique : « la bêtise de la musique » selon Milan Kundera », Recherches \& Travaux [En ligne], 78 | 2011, mis en ligne le 15 novembre 2012, consulté le 08 septembre 2020. URL : http://journals.openedition.org/recherchestravaux/451 ; DOI : https://doi.org/10.4000/ recherchestravaux.451 
Bertrand VIBERT

Université Stendhal - Grenoble 3

Traverses I9-2I

\section{D'un humanisme anti-lyrique ${ }^{\mathrm{r}}$ : "la bêtise de la musique» selon Milan Kundera}

"La bêtise n'est pas mon fort.»

La Soirée avec Monsieur Teste, Paul Valéry

Si l'histoire de la musique a pu être écrite au gré des réactions ferventes ou hostiles qu' elle a suscitées, j'ouvrirai mon propos par une brève mise en perspective. Elle aura le statut d'un rappel, puisque j'en emprunte les termes à la contribution de Timothée Picard placée au seuil de ce volume. Milan Kundera, bien qu'il ne soit pas cité, appartient précisément au groupe d'«écrivains et philosophes" de la fin du XIX ${ }^{e}$ siècle et du $\mathrm{XX}^{\mathrm{e}}$ siècle qui font preuve d'un "amour contrarié pour la musique» susceptible de se transformer en "haine apparente ${ }^{2}$. Et Timothée Picard de citer Valéry, Nietzsche, Thomas Mann, plusieurs écrivains autrichiens contemporains dont Thomas Bernardt, et bien sûr Pascal Quignard. À cette liste, il conviendrait peut-être d'ajouter Robert Musil, qui retournait la question «Y a-t-il une musique bête?» en une autre plus troublante : «La bêtise serait-elle musicale? » Or il me semble

I. Sur l'anti-lyrisme et l'anti-humanisme chez Kundera, on lira, dans des perspectives différentes de la mienne, les excellentes contributions de V.Sauzon et S. Chaudier d'une part, de M.-O. Thirouin d'autre part : respectivement "L'anti-lyrisme et ses enjeux» et "La tentation de l'anti-humanisme dans l'œuvre de Kundera", dans M.-O. Thirouin, M. Boyer-Weinmann (dir.), Désaccords parfaits. La réception paradoxale de l'ouvre de Milan Kundera, Grenoble, ELLUG, 2009.

2. Voir plus haut, p. I4.

3. Voici la réponse : «Répétitions prolongées, insistance obstinée sur un même motif, délayage des trouvailles, mouvement en rond, variations limitées sur ce qu'on a saisi à un moment donné, pathétique et véhémence tenant lieu d'illumination spirituelle : sans immodestie, la musique aurait le droit de prétendre que ce sont là ses particularités favorites.» («De la bêtise», cité par A. Roger, Gallimard, coll. «Bibliothèque des idées", 1988, p. 189, qui insiste à la suite de C. Rosset sur le caractère «tautologique» de la musique.) Voir plus loin «Un petit garçon en extase». 
que Milan Kundera est un jalon particulièrement important et significatif de cette tendance. Comme chacun des autres auteurs cités, il peut mériter une étude à part, qui sera esquissée ici.

Le dernier point de l'exposé précité examinait comment un discours sur la musique - d'amour et/ou de haine - "peut devenir matière à fiction ${ }^{4} »$. C'est dire qu'il engage une pratique d'écrivain (qui n'est pas purement spéculative, même lorsque le propos prend des allures plus ou moins théoriques). En somme - et j'y reviendrai -, parler de la musique pour un écrivain (que ce soit dans un roman ou un essai), c'est presque toujours déjà entrer en littérature, en fiction, mettre en jeu un imaginaire du langage et de l'art lui-même. La musique est ainsi un des objets privilégiés - sinon le seul possible - que la littérature se donne pour se penser et s'inventer elle-même.

\section{Dépit amoureux}

Tout lecteur tant soit peu familier de l'œuvre de Milan Kundera sait quelle place primordiale la musique occupe dans les romans et les essais, où elle peut servir la réflexion sur le roman, l'esthétique ou l'histoire de l'art. Les essais en particulier, à partir des Testaments trahis (1993), vont développer une méditation sans cesse reprise sur Stravinsky et Janáček - compatriote né à Brno comme Milan Kundera, qui fait preuve à son égard d'une tendresse toute particulière. De fait, ses essais sont autant consacrés à la musique qu’à la littérature (et à l'occasion à la peinture), à l'enseigne commune de la modernité esthétique.

Il importe de préciser que le père de Milan Kundera fut compositeur, et que lui-même a reçu une solide formation musicale, qui lui a permis de se livrer à des essais de composition. Dans les romans comme dans les essais, l'auteur de L'Insoutenable Légèreté de l'être ou des Testaments trahis illustre ainsi volontiers son propos en montrant l'exemple d'une portée musicale, parfois représentée par un dessin autographe. Et sans être jamais rebutants pour le lecteur, ses commentaires manifestent des compétences techniques. En somme, on peut présenter Milan Kundera comme un grand connaisseur et un amoureux de la musique, laquelle, si l'on en croit le discours de l'œuvre, fut pour lui un don transmis par un père admiré et, qui plus est, aimé. En retour, il est un écrivain qui confere à la musique un statut privilégié car elle lui donne à penser, que ce soit de manière fictionnelle ou non.

Pourtant, sa relation à la musique n'en est pas moins complexe et ambivalente. C'est d'abord celle, radicalement négative, que Kundera entretient

4. Voir plus haut, p. 30 et suiv. 
avec le monde moderne, - qu'il ne faut pas confondre avec la modernité esthétique. Mais, même si celle-ci est nettement valorisée, la musique et le roman - considéré comme «art » et non comme genre - y entrent en relation d'opposition dialectique, voire de rivalité. Pour nous, le problème se complique du fait que Kundera essayiste semble toujours son meilleur commentateur. Au fond, y a-t-il plus à dire que ce qu'il développe lui-même avec brio dans sa réflexion sur l'art? Peut-être - du moins faut-il tenter d'en soutenir la gageure -, car si les thèses générales sont claires, elles ont tendance à neutraliser, par leur clarté même, l'imaginaire, voire la construction du «mythe personnel» dont elles procèdent ${ }^{5}$, et à masquer le cas échéant les contradictions relatives dont elles sont tissées : en somme ce qu'elles veulent dire, qui importe en dernier ressort sur un plan littéraire, c'est-à-dire fictionnel.

Convient-il de parler de haine de la musique à propos de Milan Kundera? Quitte à moduler l'expression, je retiendrai plutôt l'horreur de sa bêtise (qui serait aussi le propre de l'homme). Sans doute, la critique de la musique - d'une certaine musique - est parfois chez lui sans appel, quoiqu'elle puisse aussi s'établir de manière plus subtile et différentielle7. Il n'empêche que la notion la plus englobante reste celle de sa "bêtise", qui dicte sa loi presque fatale dans le monde où nous vivons. Ainsi, parler de "bêtise de la musique», c'est soumettre le jugement esthétique à un jugement moral, que celui-ci soit porté contre l'individu ou la collectivité. La musique bête, ce serait en première approche celle qui favorise l'illusion sur nous-mêmes, à savoir l'aspiration kitsch à l'idylle qui est au cœur de chacun de nous, en alliée inconditionnelle du sentimentalisme et des prétentions de l'ego. C'est pourquoi le terme de «bêtise» vient naturellement sous la plume de Kundera lorsqu'il définit le kitsch : "Le kitsch, c'est la traduction de la bêtise des idées reçues dans le langage de la beauté et de l'émotion ${ }^{8}$." Bien sûr, la musique n'est qu'un exemple, mais particulièrement symptomatique de cette bêtise générale qui semble déferler sur le monde moderne.

Ces prolégomènes établis, il faut en premier lieu préciser les conditions d'une musique bête: mauvaise musique, voire antimusique, dont la réalité massive permet de poser les problèmes. Une deuxième étape plus brève - que

5. L'expression est ici entendue dans un sens général, et sans référence précise à l'approche psychanalytique de Charles Mauron.

6. La formule est très $\mathrm{XIX}^{\mathrm{e}}$ siècle, mais au fond, Milan Kundera appartient à cette lignée d'écrivains hypersensibles qui, comme Flaubert, tentent d'ériger leur œuvre en rempart contre la bêtise du monde.

7. À propos de Beethoven, par exemple, selon qu'il est considéré selon son génie propre - notamment à propos de la variation - ou en tant qu'il appartient à une période musicale dans laquelle l'écriture harmonique a supplanté le contrepoint.

8. L'Art du roman, Gallimard, coll. «NRF», I986, p. 198. 
Milan Kundera ne distingue pas explicitement de la première -, consistera à traquer la bêtise au sein d'un art musical authentique, quitte à lui trouver des parades dans la musique elle-même. On pourra alors envisager l'œuvre de Kundera comme un adieu nostalgique à la musique, dont le bénéfice peu mince est de promouvoir cet "art du roman» auquel l'auteur de $L a$ Plaisanterie (1967) n'a cessé d'être fidèle.

\section{Bête et méchante}

\section{Indignité}

La musique en tant qu'art ou phénomène culturel est un fait proprement humain. Et pourtant - tel est le paradoxe posé par Kundera -, dans certaines de ses manifestations elle porte atteinte à la dignité de l'art, et donc à celle de l'homme lui-même : ce qui est autre chose et plus que de la juger mauvaise. Or, dans la tradition romantique qui fait de la musique un absolu divin, c'est plutôt l'homme qui serait potentiellement indigne de la musique. Parlant de la bêtise, Kundera nous amène donc à renverser le point de vue en ramenant la musique à sa dimension profondément humaine.

Le chapitre I8 de la sixième partie du Livre du rire et de l'oubli', intitulée "Les anges», nous livre la réflexion inaugurale de Milan Kundera sur «la bêtise de la musique». L'expression est proférée au prix d'un grand effort par le père compositeur, devenu presque aphasique dans la toute fin de sa vie : celui-ci montre alors du doigt un haut-parleur qui diffuse de la musique et des chansons censées exprimer la joie de vivre dans la Tchécoslovaquie communiste. Nous sommes donc en présence d'un passage que Kundera qualifie d' "essai spécifiquement romanesque ${ }^{\mathrm{IO}}$ ", bien qu'il se double ici d'une dimension narrative "autobiographique ${ }^{\mathrm{II}}$ » et poignante. Le récit met ici en scène une parole testamentaire qui apporte une révélation au fils romancier, et par son truchement au lecteur. Or, dans le contexte du roman, où «les anges» représentent le conformisme idéologique (en particulier dans la Tchécoslovaquie communiste), la musique participe d'une adhésion à l'ordre des choses (y compris politiques) : elle exprime cette joie obligatoire, ce devoir de réjouissance et d'adhésion à la vie qui caractérisent le régime au prix d'un art stéréotypé dont le chanteur Karel Klos est le héraut adulér ${ }^{12}$. Or, la bêtise

9. Le Livre du rire et de l'oubli, [1978], Gallimard, coll. «Folio», I985, p. 290-293.

Io. L'Art du roman, op. cit., p. I03.

II. La visée du propos n'est en revanche nullement autobiographique, et reste globalement romanesque.

I2. Étrangement, je n'ai rien trouvé sur le chanteur Karel Klos : il faudrait s'interroger à ce sujet, et peut-être interroger Kundera lui-même, d'autant plus que ce qu'il en dit semble 
de la musique comme "simple accord avec l'être» formule très exactement ce qui trouvera sa définition dans le roman suivant, L'Insoutenable Légèreté de l'être : à savoir «le kitsch». Telle est la définition fondamentale, à la fois morale, esthétique, et métaphysique de la bêtise, à laquelle participe la musique : un art mensonger qui triche avec le réel pour en produire une vision non problématique, qui satisfait à peu de frais l'aspiration universelle à l'harmonie.

Mais au passage, dans la tentative d'interprétation par le fils de la parole d'autorité du père, on assiste à la construction d'une fable, ou d'un mythe propre au romancier :

Que voulait-il dire par là? Voulait-il insulter la musique qui était la passion de sa vie? Non, je crois qu'il voulait dire qu'il existe un état originel de la musique, un état qui précède son histoire, un état d'avant la première interrogation, d'avant la première réflexion, d'avant le premier jeu avec un motif ou un thème. Dans cet état premier de la musique (la musique sans pensée), se reflète la bêtise consubstantielle à l'être humain ${ }^{13}$. Pour que la musique s'élève au-dessus de cette bêtise première, il a fallu l'immense effort de l'esprit et du cœur, et ce fut une courbe splendide qui a surplombé des siècles d'histoire européenne et s'est éteinte au sommet de sa trajectoire comme la fusée d'un feu d'artifice ${ }^{14}$.

On voit que le mythe s'exprime ici en deux temps. D'abord, la conception d'une musique brute ou originelle, précédant l'histoire de la musique, et avec laquelle l'ère que nous vivons renouerait par une forme de soudaine régression. Or, si cette formulation définit bien la relation de Kundera à la musique et son histoire, elle ne nous dit rien en revanche qui puisse recevoir la caution d'une vérité. L'idée, dans un deuxième temps, est rendue poétiquement sensible dans une phrase à la cadence remarquable, qui impose l'évidence de sa beauté, mais qui ne doit pas non plus être confondue avec une vérité de

correspondre parfaitement à l'autre Karel : chanteur adulé en Tchécoslovaquie et en Allemagne, avant la chute du mur et après, Karel Gott est le type même de l'artiste kitsch, consensuel et inoxydable, par définition du côté du pouvoir en place. Sur le chanteur et sur le musée qui lui est consacré de son vivant, voir Gottland de Mariusz Szczygieł [2006], récits traduits du polonais par Margot Carlier, Actes Sud, 2008. On peut aussi se reporter par exemple à son interprétation de «Popelka» sur "You Tube»: http://www.youtube.com/ watch ?v=2FCVfq4dy_o (site consulté le 30-09-2009).

I3. Pour une analyse critique de la formule de M. Kundera, voir A. Roger, Bréviaire de la bêtise, op. cit., p. 40-42, qui lui préfère celle de Gombrowicz alléguant une «bêtise consubstantielle à la raison ", dans laquelle il voit la solution au problème philosophique de l'antinomie de la bêtise. A. Roger part d'une critique de "la raison suffisante», jouant sur le double sens de l'adjectif. Cette bêtise de la raison rejoint néanmoins celle des "paradoxes terminaux" énoncés au début de L'Art du roman, op. cit. De même, la bêtise fondamentale de l'ego consonne avec l'œuvre de M. Kundera même si - différence de taille -, A. Roger l'impute à la raison quand $M$. Kundera la met au compte du sentiment érigé en valeur.

I4. Le Livre du rire et de l'oubli, op. cit., p. 290-29I. 
discours. Cette fable nostalgique, c'est celle d'une mort en beauté donnée comme déjà accomplie ${ }^{15}$. Il n'en reste pas moins que Milan Kundera entend opposer une musique valorisée - fruit de l'intelligence et de la sensibilité - à la sentimentalité facile de la musique des radios et des haut-parleurs. À cet égard, le rock, la musique pop et les guitares reviendront de façon obsessionnelle dans la prose des essais et des romans pour traduire le degré extrême du dévoiement musical, comme si Milan Kundera était resté délibérément sourd à tout ce qui est venu après, au risque de faire sourire les jeunes gens qui ont aujourd'hui 20 ans. Pourquoi tant de haine?

On peut y voir d'abord le mépris de l'homme de haute culture musicale pour les genres médiatiques et spectaculaires de la musique (pop, rock et variété), d'autant plus qu'ils seraient portés par une jeunesse qui affirme ainsi - c'est la signification profonde du tapage - des valeurs majoritaires ou consensuelles : tout ce qu'il faut, en somme, pour susciter l'ire et l'ironie de Kundera. Mais il convient aussi de faire la part de l'exigence artiste dans l'invention, la richesse et la complexité des formes, dont témoignent dans l'œuvre les développements sur la musique. C'est là une autre manière de revendiquer une forme d'élitisme ${ }^{16}$. Troisième argument, celui qui tient à une conception élaborée du "rythme», la scansion régulière étant au contraire associée à la bêtise : «L'assommant primitivisme rythmique du rock : le battement du cour est amplifié pour que l'homme n'oublie pas une seconde sa marche vers la mort ${ }^{17}$.» À quoi Kundera oppose la musique savante, de Beethoven à Messiaen, conçue comme effort pour s'affranchir de la mesure et de la scansion simple du rythme. Mais cette fois, l'argument achoppe sur ce que dit Kundera ailleurs, et ressemble fort à un brillant paradoxe servi ad hoc. Car partout dans l'œuvre, c'est le kitsch qui est stigmatisé, précisément parce qu'il est un voile posé devant ce que la vie humaine a d'insupportable, c'est-à-dire in fine la mort ${ }^{18}$. Le kitsch trahit ainsi notre manière bête et vulgaire de nous soustraire à la réalité par le biais d'une expérience esthétique aussi intense que douteuse. Et pour le coup, la pop et le kitsch peuvent faire cause commune dans la pensée de Kundera.

15. Celle-ci n’a donc rien à voir avec «l'art de mourir en beauté» par lequel Verlaine définit la décadence.

I6. Voir à ce sujet l'entrée "Élitisme» dans L'Art du roman parmi les "Soixante et onze mots» qui constituent le dictionnaire personnel de Kundera (op. cit., p. 157). Il constate l'apparition récente du mot (autour de 1968) tant en France que dans les pays communistes, avec un "éclairage de négativité sinon de mépris», et qui plus est réservé à la stigmatisation de l'élite culturelle. Il y voit le signe de la promotion d'autres élites, policière à l'est et massmédiatique à l'ouest.

17. L'Art du roman, op. cit., p. I8I.

18. À titre d'exemple de musique bête jusqu'au grotesque, on lira le passage où la danse est associée à la musique dans l'île des enfants du Livre du rire et de l'oubli, op. cit., p. 302 et suiv. 


\section{Facilité}

Dans Les Testaments trahis, Milan Kundera se met en scène dans une anecdote fournie par un souvenir d'enfance, qu'il intitule «Un petit garçon en extase». Le petit garçon, c'est lui, se livrant au piano à des «improvisations passionnées" mais sommaires, composées de deux accords et d'un «motif mélodique primitif», vivant ainsi un paroxysme d'émotion qui est aussi une expérience d'aliénation volontaire ${ }^{19}$. Dans un essai cette fois, la figure du père revient - et intervient - pour faire comprendre le sens de ce qui a été vécu :

(Une fois, mon père, musicien, tout furieux - je ne l'ai jamais vu furieux ni avant ni après - accourut dans ma chambre, me souleva du tabouret et me porta dans la salle à manger pour me déposer, avec un dégoût à peine dominé, sous la table ${ }^{20}$.)

La parenthèse, qui est comme une concession discrète à la narration autobiographique, ne doit pas nous tromper : sous couvert de simple récit, elle est évidemment un argument d'autorité, par le geste sans appel qu'elle exprime à l'intention de l'enfant et du lecteur. La loi du père, on se souvient que c'est ce qui manque à Jaromil, le poète lyrique et communiste de La vie est ailleurs ${ }^{21}$. Partout dans l'œuvre, l'ivresse ou l'extase des sentiments suscite des réactions de dégoût, voire de répulsion devant quelque chose d'impudique et d'obscène $^{22}$. Suit une critique radicale de l'extase, définie comme "identification absolue à l'instant présent, oubli total du passé et de l'avenir». Au fond, dit Kundera, il est une "extase quotidienne, banale, vulgaire»(p. Io6) qui n'a rien à voir avec la mystique, et qui, là encore, porte atteinte à l'humanité de l'homme. Si l'extase individuelle est déjà suspecte, celle du groupe, de la collectivité le sera bien davantage encore. Ainsi, toutes les foules en extase que réunit la musique, le sport ou la politique - cette dernière dans les "cortèges" de L'Insoutenable Légèreté de l'être ${ }^{23}$-, sont en proie au kitsch. Toutes les communions et adhésions sans distance sont pernicieuses, parce qu'elles chantent l'air mensonger de la grande Idylle. On comprend que la musique soit un de ses véhicules privilégiés. Pourtant, par une terrible ironie, c’est transformée en bruit que la musique fait intrusion dans le monde moderne.

19. Chez le Quignard de La Haine de la musique [1996], Gallimard, coll. «Folio», 1997, l'aliénation est au contraire pensée comme involontaire : voir le VII ${ }^{\mathrm{e}}$ traité du même nom.

20. Les Testaments trahis, Gallimard, coll. "Folio», I993, p. I04.

21. La vie est ailleurs [1973], Gallimard, coll. "Folio", I987.

22. Voir, dans L'Ignorance [2000], Gallimard, coll. «NRF», 2003, Josef retrouvant un journal d'adolescent et reniant le "morveux" qui lui paraît complètement étranger à lui-même (p. 8I).

23. L'Insoutenable Légèreté de l'être [1984], Gallimard, coll. «Folio», I989, passim. 


\section{Brutalité}

Que signifie le fait que le bruit et la musique soient devenus le lot commun des citadins modernes (cette fois à l'ouest ou dans le monde postcommuniste)? Pour Milan Kundera, la banalisation et la généralisation d'une forme spéciale de violence. Car celle-ci traduit aussi une véritable lutte des ego en vue de l'occupation d'un territoire symbolique. Au début de L'Immortalité, Agnès est assaillie par le «vacarme atroce» de la ville moderne ${ }^{24}$, bruits et musiques confondus : c'est une jeune fille sur une moto dont le pot d'échappement a été enlevé et dont le bruit paraît l'expression même du moi de la jeune fille imposé à tous; mais c'est aussi une fugue de Bach échappée d'une fenêtre ouverte qui, au lieu de résister au bruit assourdissant des marteaux-piqueurs et des voitures, contribue à l'agression sonore généralisée (p. 39-42). Plus généralement, Kundera intente le procès de la musique devenue bruit ${ }^{25}$ : la violence sonore, et la laideur sonore qui est elle-même une forme de violence, semblent ainsi définir les conditions fondamentales de la vie citadine moderne.

Plusieurs critiques ici se superposent, qui définissent les conditions dans lesquelles la musique devient bruit. C'est d'abord, ipso facto, le cas de toute musique imposée (même une fugue de Bach pour qui aspire légitimement au silence). Dans le cas précité, elle participe du vacarme général, que le texte nomme à son tour «fugue» par dérision. À quoi il faut ajouter que la musique en continu est négation de la musique, par le seul fait qu' elle méconnaît le silence, qui est à la fois sa condition et sa respiration. Troisième argument : une musique qui n'est pas une œuvre - musique d'ambiance ou $m u z a k^{26}-$ est par essence du bruit : venant de personne, elle ne construit rien et ne s'adresse à personne : sur le plan esthétique, elle est un message proprement nul (et non avenu). Dernier argument, où affleure plus nettement la sensibilité, voire la susceptibilité d'artiste de Milan Kundera : la médiatisation de la musique dans un flux indifférencié constitue la mise à mort la plus efficace qui soit des compositeurs qui eurent à cœur de créer des œuvres. Il s'agit

24. L'Immortalité [1984], Gallimard, coll. «Folio», I993, p. 40.

25. L'Ignorance [2000], Gallimard, coll. "NRF», 2003, p. I36-137 et passim. À opposer à "Musique et bruit", dans Les Testaments trahis, op. cit., p. 85 et suiv., où la dialectique des termes est mise au service de la musique elle-même.

26. Passée dans le langage courant en Amérique du Nord, la muzak est une forme de musique aseptisée, mise aux normes (les passages de niveau sonore très forts ou très faibles en sont nivelés), parfois diffusée dans les ascenseurs, sur les lignes d'attente des standards téléphoniques ou dans les supermarchés. Ce terme est une antonomase du nom de la compagnie Muzak (mot-valise formé à partir de «music» et «Kodak»), qui fut pionnière dans ce domaine (dans les années 20). Voir http://fr.wikipedia.org/wiki/Muzak (site consulté le IO septembre 2009). 
alors, non pas tant de "testaments trahis» que - d'une manière plus tristement dérisoire - de testaments brouillés et rendus illisibles. C'est ainsi que, selon Milan Kundera, la noble rivalité entre Schönberg et Stravinsky fit sousestimer au premier la "peste musicale» propagée par les médias modernes, qui étaient pourtant leur ennemi commun, bien plus insidieux et redoutable que le jugement de la postérité qui les aurait départagés :

[...] l'avenir, ce fut le fleuve, le déluge de notes où les cadavres des compositeurs flottèrent parmi les feuilles mortes et les branches arrachées. Un jour, le corps mort de Schönberg, ballotté par les vagues démontées, heurta celui de Stravinsky et tous les deux, dans une réconciliation tardive et coupable, continuèrent leur voyage vers le néant (vers le néant de la musique qu'est le vacarme absolu ${ }^{27}$ ).

Manière inimitable de Kundera, chaque fois qu'il entreprend d'exposer une idée, de raconter une histoire ou même de construire un mythe, fût-il de la fin plutôt que de l'origine. On peut en tout cas juger de son sens particulier de la dramatisation, qui recourt, pour traduire l'histoire de la musique parvenue à son terme d'oubli, à la métaphore filée de la catastrophe, du fleuve en crue et des flots démontés, tout à la fois irrémédiablement destructeurs et niveleurs.

Sans doute, l'idée selon laquelle notre monde contemporain serait envahi par une musique ayant renié les valeurs essentielles de l'art musical est peu originale en elle-même. Pour qu'elle soit précieuse, il faut, comme chez Kundera, qu'elle porte la marque d'un style et d'un imaginaire qui lui confèrent une signification particulière au regard de l'œuvre elle-même. Mais audelà, il est un autre territoire où la musique, même potentiellement bonne ou belle, inspire la défiance, ce qui est à la fois plus intéressant et plus scandaleux pour la pensée : ce territoire, c’est le lyrisme.

\section{Bonne et bête?}

\section{Grands sentiments}

L'œuvre de Milan Kundera se caractérise globalement par un refus viscéral de tout lyrisme ${ }^{28}$, entendu dans la double acception d'une attitude existentielle et d'une définition particulière de la poésie comme genre. Les deux sont

27. L'Ignorance, op. cit., p. I37.

28. À lire surtout le dernier essai paru à ce jour, Une rencontre, Gallimard, coll. "NRF", 2009, ce jugement demande pourtant à être nuancé. J'essaye de m'en expliquer ailleurs : voir "Nostalgie de l'“être oublié"; dégoût de "l'accord catégorique avec l'être": des enjeux romanesques d'une contradiction", dans Milan Kundera. Ce que peut la littérature, actes du colloque international de Brno, 28-30 mai 2009, à paraître. 
magistralement réunis dans la figure du poète Jaromil, le triste héros de $L a$ vie est ailleurs (1973). En ce sens, le lyrisme quitte la musique pour occuper de façon privilégiée le terrain de la poésie, particulièrement sollicitée comme on sait dans les pays communistes. Cette haine du lyrisme en tant que valeur s'enracine en effet dans l'expérience totalitaire du bloc de l'est qui en avait fait d'abord un impératif politique et idéologique :

Plus que la Terreur, la lyrisation de la Terreur fut pour moi un traumatisme. À jamais, j’ai été vacciné contre toutes les tentations lyriques. La seule chose que je désirais alors profondément, avidement, c'était un regard désabusé. Je l'ai trouvé enfin dans l'art du roman ${ }^{29}$.

Tout lyrisme risque donc de verser dans le kitsch, et de s'alimenter à la même source que le kitsch. Mais en tant qu'attitude existentielle, il peut aussi bien caractériser tous les arts, à commencer par la musique dont il est issu et qu'il caractérise pour partie. Une fois les idéologies vaincues, l'imagologie triomphante prendra le relais ${ }^{30}$, et le kitsch pourra se métamorphoser sans rien perdre de sa puissance. Étant partout, il peut aussi retrouver son lieu d'origine, et la musique ne le cède désormais en rien à la poésie. Ainsi, à l'entrée du mot «kitsch» du petit dictionnaire personnel établi par Milan Kundera dans L'Art du roman, les exemples musicaux s'imposent naturellement ${ }^{3 \mathrm{I}}$. Au passage, l'auteur explique pourquoi le romantisme sentimental, largement dominant dans l'esthétique du centre de l'Europe au XIX ${ }^{\mathrm{e}}$ siècle, a suscité dans l'élite intellectuelle une réaction de rejet inconnue en France, bien davantage marquée par l'héritage de l'esthétique réaliste. Et c'est, semble-t-il, une fois émigré en France que Kundera s'intéresse à une attitude lyrique rapportée directement à la musique : je pense en particulier à L'Immortalité, ce premier grand roman complètement situé dans l'orbite française (bien qu'il soit encore écrit en tchèque). Ainsi, à l'intention d'un public d'abord français et occidental, le personnage de Laura, bien que musicienne manquée, semble un peu le pendant musical de ce que Jaromil était à la poésie : comme le héros de La vie est ailleurs, elle incarne à sa manière les dangers du lyrisme, mais dans un tout autre contexte (historique et géographique, politique, social, culturel). Avec elle, Milan Kundera met en scène l'emphase des grands sentiments, qui ne le sont qu'à la mesure - ou à la démesure - de l'ego, n'étant jamais dus à une

29. Les Testaments trahis, op. cit., p. I9I.

30. Voir L'Immortalité, op. cit., p. I7I et suiv. L'imagologie, pour dire vite, est le règne aveugle et sans partage des médias soustraits à toute raison, selon l'unique loi de la valorisation de l'instant émotionnel, au gré d'un continuel changement dicté par la nécessité d'occuper tant l'espace physique que symbolique (celui des esprits). Après la fin du communisme, elle est la métabolisation planétaire du kitsch.

3I. Voir «Soixante et onze mots», L'Art du roman, op. cit., p. I65. 
inspiration noble ou authentiquement altruiste. C'est dire si l'appréciation de la grandeur reste purement quantitative.

S'agissant de valeurs, la caractérisation du lyrisme romantique se soucie peu d'une stricte périodisation. Elle inclut encore Wagner, à l'égard duquel Milan Kundera n'est pas tendre : la «mélodie infinie» devient chez lui, au prix d'une belle trouvaille d'irrévérencieuse drôlerie, «le bâillement infini de la mélodie wagnérienne ${ }^{32} »$. C'est que précisément on ne trouve guère de distance, d'humour, et moins encore d'autodérision chez le maître de Bayreuth. Et assurément, il y a quelque chose de rédhibitoire dans ce qui ne sera jamais esthétiquement, pour l'auteur de Risibles amours, que «la pompe du lyrisme». Je soupçonne à cet égard que Tristan et Isolde, que Milan Kundera se garde d'évoquer jamais, doit être le parangon de ce qu'il déteste en fait de grande musique lyrique - au double sens du terme -, bien que chef-d'œuvre peu contestable par ailleurs. Mais c'est plutôt un certain Mahler - celui de l'adagietto de la Cinquième Symphonie popularisé par Mort à Venise de Visconti $\left(197 \mathrm{I}^{33}\right)$ ? - qui fait les frais, dans L'Immortalité, d'une conception hautement ironique : "La musique : une pompe à gonfler l'âme» (p. 305). Il faut faire ici la part de l'outrance provocatrice et d'une mauvaise foi assumée avec jubilation. : Milan Kundera sait bien, à l'encontre de son personnage Laura et même de son narrateur, que Mahler ne saurait être cantonné dans la figure $\mathrm{du}$ « dernier grand compositeur qui s'adresse encore naïvement et directement à l'homo sentimentalis" (p. 306). Mais il y a plus. Car telle qu'elle est, la formule a quand même quelque chose à nous apprendre : Mahler représente sans doute l'apogée du lyrisme symphonique, son horizon indépassable dans l'histoire de la musique. Surtout, comme le montre à l'envi le personnage de Laura, la musique n'est pas seule en cause, tant il est vrai que sa prétendue bêtise doit beaucoup à l'auditeur, qui n'a de cesse d'en rajouter.

\section{Contagion musicale}

Kundera revient à diverses reprises sur l'argument en lui donnant le tour d'une anecdote à caractère très général (et donc inventée) que je voudrais d'ailleurs contester :

Un Stravinsky a beau récuser la musique comme expression des sentiments, l'auditeur ne sait pas la comprendre autrement. C'est la malédiction de la musique, c'est son côté bête. Il suffit qu'un violoniste joue les trois premières longues

32. Je n’ai pu retrouvé la référence de la citation, citée de mémoire, que je ne saurais pourtant me flatter d'avoir inventée.

33. Circonstance sans doute aggravante aux yeux de Milan Kundera, il s'agit d'une adaptation de la célèbre nouvelle de Thomas Mann du même nom (I9I2), adaptation qu'on peut en outre juger particulièrement frelatée : les testaments sont donc doublement trahis. 
notes d'un adagio pour qu'un auditeur sensible soupire : «Ah, que c'est beau!» Dans ces trois premières notes qui ont provoqué l'émotion, il n'y a rien, aucune invention, aucune création, rien du tout : la plus ridicule «duperie sentimentale». Mais personne n'est à l'abri de cette perception de la musique, de ce soupir niais qu'elle suscite ${ }^{34}$.

Il y a là, à mon sens, un autre exemple de mauvaise foi tout à fait consciente, qui sert à nouveau un argument provocateur et d'une certaine manière imparable. C'est que le raisonnement, s'il est intellectuellement séduisant, est de surcroît intimidant, car il établit un rapport de forces qui en impose au lecteur : celui-ci est enclin à se percevoir comme moins averti que l'auteur, et peut-être même coupable de sentimentalisme musical. Or l'établissement de la preuve s'en tient à la généralité de l'exemple, auquel l'auteur se garde bien de fournir la moindre illustration précise. À titre de compensation, le sophisme - car c'en est un - se doit d'être brillant et péremptoire. Et pour le coup, je serais presque tenté de dire que Milan Kundera fait le bête, car il sait mieux que personne que la question ne se pose pas exactement dans ces termes-là : les notes ne sont pas toutes seules, elles sont portées par un son et par la présence d'un interprète, elles sont éventuellement accompagnées, harmonisées, elles naissent sur un fond particulier de silence, elles ont été amenées par le mouvement précédent, etc. Pourtant Kundera s'obstine, puisqu'il récidive à plusieurs reprises dans les essais et l'œuvre romanesque depuis les années quatre-vingt. On retrouve encore le même argument dans son dernier essai, qui reprend et commente un texte antérieur. Dans L'Immortalité en revanche, avant de formuler l'argument des trois notes, d'ailleurs réduites à deux (p. 305), le narrateur-auteur commençait par célébrer le caractère unique de l'héritage musical européen : "ce miracle qu'est l'histoire millénaire de la musique européenne, avec toute sa richesse de formes et de styles!» (ibid.) Mais il signalait aussitôt le danger lié à son ambivalence et à l'héritage dont nous sommes tributaires : à défaut certes $\mathrm{du}$ sentiment lui-même, la valorisation $d u$ sentiment ${ }^{35}$ du moins serait un attribut inhérent à la musique européenne. Or, nous avons vu que, dans le contexte particulier du totalitarisme où le sentiment a été mis au service de fins politiques perverses, le lyrisme est devenu définitivement insupportable. Sur le plan esthétique cependant, les compositeurs "modernes» ont apporté une réponse en contestant le lyrisme, autant dire l'expression et l'expansion de la subjectivité issue du romantisme.

34. Une rencontre, op. cit., p. 95.

35. Voir L'Immortalité, ibid., p. 289, la définition de "l'homo sentimentalis» comme celui, non "qui éprouve des sentiments», mais «qui les a érigés en valeurs». 
Antidote moderne

On comprend bien dès lors les raisons pour lesquelles Milan Kundera n'a cessé de rendre des hommages appuyés à Janáček, Stravinsky ou Schönberg. Pour s'opposer à la lyrisation de la terreur, c'est en particulier à Bartók, Varèse et à Xenakis qu'il fait appel, d'une façon systématique qui semble faire pendant à l'argument des premières notes du violoniste s'adressant à notre bêtise sentimentale. Mais cette fois, il évoque un souvenir personnel précis et beaucoup plus convaincant. Dans la période qui a suivi l'invasion de la Tchécoslovaquie par les troupes russes, Milan Kundera dit être «tombé amoureux" de Varèse et de Xenakis dont les univers musicaux lui ont paru salutaires, précisément parce qu'ils inventaient des sonorités objectives qui ne laissaient aucune place à l'expression de cette subjectivité humaine qu'il trouvait dévoyée dans le lyrisme politique. Déjà, sans être aussi radicale que celle de Xenakis, la musique de Bartók permettait d'accéder à une forme de beauté objective dans la mesure où, tout en laissant une place à la subjectivité, elle lui interdisait l'épanouissement de la moindre complaisance sentimentale. Le problème se trouve ainsi posé dans toute sa rigueur :

[...] je pense à l'adagio du troisième Concerto pour piano et orchestre de Bartók [...]. Le thème hypersubjectif d'une ineffable mélodie alterne ici avec l'autre thème hyperobjectif $[\ldots]$ : comme si le pleur d'une âme ne pouvait être consolé que par la non-sensibilité de la nature. (Les Testaments trahis, p. 87-88.)

Quinze ans plus tard, relatant la même expérience, Kundera ajoute :

C'est alors que la musique m'est apparue comme le bruit assourdissant des émotions, tandis que le monde des bruits dans les compositions de Xenakis est devenu beauté; la beauté lavée de la saleté affective, dépourvue de la barbarie sentimentale ${ }^{36}$.

Le double renversement - musique devenue bruit d'un côté, monde des bruits devenant beauté de l'autre - est indéniablement efficace, d'autant plus qu'il débouche sur un double oxymore chargé de miner la valeur a priori consensuelle de l'affect ou du sentiment : «saleté affective», "barbarie sentimentale». Ainsi, il est une place pour l'humain au-delà de la subjectivité. En d'autres termes, il convient de dissocier la valeur attribuée à l'humain du degré de subjectivité qu'on lui prête. Pour Milan Kundera en tout cas, l'humanisme ne saurait être qu'antilyrique. Mais on l'a compris : la musique pour Kundera a déjà émis son chant du cygne, sa possibilité de création et de développement en tant qu'art étant parvenue à son terme. C'est pourquoi le roman sera la meilleure réponse esthétique à la bêtise de la musique.

36. Une rencontre, op. cit., p. 96. 
Recherches \& Travaux $-\mathrm{n}^{\circ} 78$

\section{Adieu à la musique}

\section{Épilogue}

Dans Les Testaments trahis, Milan Kundera développe un parallèle entre l'histoire de la musique et l'histoire du roman. Si, selon lui, "elles se sont déroulées à des rythmes semblables, pour ainsi dire, en deux mi-temps» (p. 73), les césures entre les deux mi-temps sont asynchrones. Pour la musique, la première période irait des débuts de la polyphonie jusqu'à L'Art de la fugue de Bach, qui peut en constituer «l'apogée symbolique» (donc jusqu'au milieu du XvIII ${ }^{\text {e }}$ siècle); pour le roman, elle irait de Rabelais et Cervantès à Laclos, Sterne et Diderot $\left(\mathrm{du} \mathrm{XVI}^{\mathrm{e}}\right.$ au XviII ${ }^{\mathrm{e}}$ siècle). Les valeurs esthétiques caractéristiques de cette première mi-temps seraient l'invention formelle, le jeu, la liberté, et en particulier pour le roman le refus de la mimèsis, tout à la fois imitation et vraisemblance. La seconde mi-temps de la musique irait du classicisme au postromantisme et verrait le règne de la mélodie (et de l'harmonie) : en gros de Mozart à Wagner et Mahler. Pour le roman, elle engloberait tout le $\mathrm{XIx}^{\mathrm{e}}$ siècle, de Walter Scott et Balzac jusqu’à Zola et Proust. Les valeurs de la seconde mi-temps seraient la mise en avant de la subjectivité, et conjointement la vraisemblance pour le roman.

Mais ce parallèle peut-être hasardeux ne prend tout son sens qu'à la fin du propos, lorsque Kundera introduit la notion d'un «épilogue», ou encore d'un éventuel "troisième temps» au-delà de la fin de l'histoire, qui serait comme un sursis mélancolique et nostalgique. Ce dernier temps met en avant «les grandes œuvres du modernisme en tant que réhabilitation de la première mitemps» (p. 9I), tandis que la seconde, nous dit Kundera, l'"a non seulement éclipsé[e] [mais] refoulée» (p. 75). Et de citer à la fois les romanciers - de Kafka à Musil, Broch et Gombrowicz jusqu'à Fuentes -, et les compositeurs "modernes», au premier rang desquels Stravinsky (l'adjectif "moderne» étant ici à la fois une étiquette descriptive et un coefficient de valeur). Voyons d'abord les romanciers :

Les plus grands romanciers de la période post proustienne [...] ont été extrêmement sensibles à l'esthétique du roman, quasiment oubliée, qui a précédé le $\mathrm{XIX}^{\mathrm{e}}$ siècle : ils ont intégré la réflexion essayistique à l'art du roman; ils ont rendu plus libre la composition; reconquis le droit à la digression; insufflé au roman l'esprit du non-sérieux et du jeu; renoncé aux dogmes du réalisme psychologique en créant des personnages sans prétendre concurrencer (à la manière de Balzac) l'état-civil; et surtout : ils se sont opposés à l'obligation de suggérer au lecteur l'illusion du réel : obligation qui a souverainement gouverné toute la deuxième mi-temps du roman ${ }^{37}$.

37. Les Testaments trahis, op. cit., p. 9I-92. 
On s'en doute, tout ce que Kundera affirme ici, en l'appliquant à d'autres, esquisse les grands traits de sa propre poétique telle qu'il l'expose ailleurs. Il s'agit donc également pour lui de se situer par une sorte de plaidoyer pro domo qui lui confère une place précaire peut-être, mais néanmoins précieuse dans l'histoire de l'art en général, et du roman en particulier : celle d'une famille d'élection. Car si à l'instar des hommes, l'art est voué à une mort programmée à plus ou moins long terme, il n'en reste pas moins que le sursis, épilogue ou troisième temps, c'est-à-dire le moment de son apogée, irradie toute l'histoire de l'art de sa beauté mélancolique. Pourtant cette conclusion ne peut revêtir que la forme d'une interrogation anxieuse et lancinante, sans autre réponse assurée qu'une «image» à la beauté émouvante :

Ne devrais-je pas corriger ma métaphore sur l'histoire de la musique et celle du roman? Ne devrais-je pas dire qu'elles se sont déroulées en trois temps?

$\mathrm{Si}$, je corrigerai ma métaphore et ce d'autant plus volontiers que je suis passionnément attaché à ce troisième temps en forme d'" embrasement du ciel à la fin du jour ", attaché à ce temps dont je crois faire moi-même partie, même si je fais partie de quelque chose qui n'est déjà plus.

Mais revenons à ma question : que signifie la volonté de Stravinsky d'embrasser le temps entier de la musique? Quel en est le sens?

Une image me poursuit : selon une croyance populaire, dans la seconde de l'agonie celui qui va mourir voit se dérouler devant ses yeux toute sa vie passée. Dans l'œuvre de Stravinsky, la musique européenne s'est souvenue de sa vie millénaire; cela a été son dernier rêve avant de partir pour un sommeil éternel sans rêves ${ }^{38}$.

Par-delà Stravinsky, c'est donc très explicitement la situation de sa propre entreprise romanesque qu'interroge Kundera. Mais qu'en est-il à ce stade du parallèle entre la musique et le roman? Jusqu'ici, l'auteur des Testaments trahis semblait le poser comme une hypothèse - et donc avec une certaine distance ${ }^{39}-$, ou encore le présenter comme une théorie toute personnelle. Or, à présent, c'est la mort de la musique que Milan Kundera met en scène de façon mélancolique, dans ce qui est déjà l'ébauche d'une fiction. Ailleurs, dans Une rencontre, Kundera dira que la peinture est morte après Francis Bacon $^{40}$. Pourquoi donc le sort du roman serait-il différent? L’art du Troisième Temps est placé de façon solidaire sous le signe de la mort prochaine et de la

38. Ibid., p. 94-95. Le "sommeil éternel sans rêves» de la musique, c'est aussi ce à quoi aspirent certains des personnages élus de Kundera - comme Agnès dans L'Immortalité -, qui cherchent à être délivrés de cette lutte entre les ego qu'est la vie sociale.

39. Voir la prétérition, ibid., p. 9I-92: "Je ne veux pas faire un parallèle facile entre le roman et la musique, les problèmes structurels de ces deux arts sont incomparables; pourtant les situations historiques se ressemblent : comme les grands romanciers, les grands compositeurs modernes (cela concerne Stravinsky aussi bien que Schönberg) ont voulu embrasser tous les siècles de la musique, re-penser, re-composer l'échelle de valeurs de son histoire; pour cela, il leur a fallu faire sortir la musique de l'ornière de la deuxième mi-temps [...].»

40. Voir Une rencontre, op. cit., p. 15-30. 
nostalgie, et pourtant Kundera ne cesse dans son œuvre de défendre la vitalité du roman en tant qu'art ouvert sur le présent du monde, - comme une revanche esthétique de la beauté et de l'intelligence sur ce que le monde est devenu. Au fond, quand il se place du point de vue général de la modernité, musique et roman se rejoignent dans leur situation d'arts. Mais quand il envisage le roman de l'intérieur, en tant que praticien, Kundera lui assure un statut privilégié, qui serait celui du premier des arts (sur le plan de sa hiérarchie personnelle) et du dernier des arts (sur le plan de son développement possible) : c'est là sans doute sa fiction, ou son moteur en tant que romancier. De ce point de vue, la réflexion de Kundera sur le roman dans sa relation à la musique n'est pas sans rappeler celle de Mallarmé qui tantôt associait musique et poésie au nom des valeurs de l'art opposées aux valeurs du monde, tantôt les opposait pour accorder à la poésie la suprématie dans la hiérarchie des arts. Mais Milan Kundera va plus loin encore puisqu'il annonce la mort de la musique.

\section{Minuit sur le cadran de la musique}

"S'il est vrai que l'histoire de la musique est finie ${ }^{4 \mathrm{I}}$ » : ainsi commençait le chapitre du Livre du rire et de l'oubli commenté plus haut. Mais c'est dans "Le refus intégral de l'héritage ou Iannis Xenakis ${ }^{42}$ » qu'on peut lire l'argument qui fonde aux yeux de Kundera l'opposition entre le romancier et le musicien modernes, entre Joyce et Xenakis :

Tout en étant "prophète de l'insensibilité», Joyce pouvait rester romancier; Xenakis, en revanche, a dû sortir de la musique [...] : pour la première fois, quelqu'un a osé dire à la musique européenne qu'il est possible de l'abandonner. De l'oublier ${ }^{43}$.

De ce point de vue, on ne refusera pas à Kundera le mérite de la cohérence : si Stravinsky se souvenait une dernière fois de l'histoire entière de la musique, Xenakis, lui, entreprend d'aller au-delà, "de l'oublier». En somme la musique est morte deux fois en tant qu'art (sans qu'il faille ici introduire une stricte chronologie) : la première, parce que la mauvaise musique a chassé la bonne, en la rendant inaudible; la seconde, parce que l'aventure de la modernité semble avoir interdit le renouvellement formel de la musique en la contraignant à sortir d'elle-même et de sa propre histoire. «S'il est vrai que l'histoire de la musique est finie», reste donc le roman, qui pourra passer pour l'avenir de la musique, à défaut de cette «musique de l'avenir» que pouvait encore prophétiser Wagner.

4I. Le Livre du rire et de l'oubli, op. cit., p. 290.

42. Le texte a été publié en 1980, puis augmenté de deux interludes en 2008, et finalement repris dans Une rencontre, op. cit.

43. Ibid., p. 97-98. 
Car la mélancolie inhérente à la conception du roman de Kundera est nostalgique d'un état du monde, d'un état de l'art et parfois aussi du roman, mais elle n'est pas un constat de la mort du roman lui-même. C'est en ce sens que celui-ci semble l'art voué à recueillir l'héritage de la musique. Sans qu'il soit utile de le développer ici, on sait que la musique sert de modèle à la composition romanesque : conception largement explicitée depuis L'Art du roman (1986), dont la "Quatrième partie», illustrée par les romans antérieurs, est centrée sur la question de la polyphonie romanesque, la variation étant l'autre loi fondamentale de la poétique de Kundera ${ }^{44}$. Si la musique fournit un modèle au roman, réciproquement le roman pourra suppléer un art désormais voué à se retourner sur son passé. Car le roman au contraire, même s'il montre la laideur ou la bêtise du monde moderne, peut continuer à explorer le chemin de sa beauté propre. Ce faisant, il prend en charge la musique, thème et objet de réflexion que vont incarner des personnages.

La relation des personnages à la musique recouvre différentes attitudes possibles. Quant au point de vue des romans sur la musique, il est le plus souvent négatif ou mélancolique. Ainsi, dans La Plaisanterie, Jaroslav, l'ami du personnage principal Ludvik, est un musicien qui essaye de perpétuer l'héritage millénaire de la musique populaire morave, malgré un dévoiement et une folklorisation opérés par le régime communiste. Quant à Ludvik, après que tout a été dévasté au terme du roman - le monde et les destinées individuelles des personnages -, il demande à Jaroslav la faveur de se joindre à son orchestre lors du concert de la fin d'après-midi et d'y tenir une partie de clarinette. Il s'agit de renouer avec un monde qu'il a lui même «autrefois déserté45", et qui lui apparâit maintenant abandonné par tous et trahi par toutes les formes de bêtise officielle - «la publicité», "la propagande politique», «les utopies sociales", "les troupes des fonctionnaires de la culture», et même "l'adhésion affectée» des gens de sa génération. Ainsi, ce monde peut désormais susciter en Ludvik l'amour et l'insoutenable nostalgie d'une fidélité qui renoue avec l'essence fragile et précieuse de valeurs perdues :

[...] cette solitude le purifiait [le monde]; [...] elle le purifiait comme quelqu'un qui n'en a plus pour longtemps; elle l'illuminait d'une irrésistible dernière beauté; cette solitude me le rendait (Ibid., p. 446-447.)

On aura reconnu, dans ce premier roman de Kundera, l'expérience fondatrice de la beauté mélancolique - précisément associée à la musique -, celle-là même qu'on a vue reconduite dans la réflexion essayiste et la méditation sur Stravinsky.

44. Voir L'Art du roman et Le Livre du rire et de l'oubli, op. cit., passim.

45. La Plaisanterie [1967], Gallimard, coll. «Folio», 1987, p. 445. 
Autre exemple remarquable, celui de Teresa dans L'Insoutenable Légèreté de l'être. Le personnage se caractérise par un désir de "s'élever», d'échapper par la littérature et la musique à l'emprise d'une mère impudique, à sa condition de serveuse et plus généralement à la vulgarité environnante. Or sa rencontre avec Tomas est placée sous le signe d'un quatuor de Beethoven (et donc de la beauté). Question intriquée avec celle, centrale dans le roman, du hasard et du destin. On ne saurait donc s'étonner qu'il s'agisse du dernier mouvement du dernier quatuor de Beethoven (op. I35, en fa majeur), sous-titré «Es muss sein» ("Cela doit être»). Dans le roman, la formule devient dès lors, et de façon récurrente, l'expression la plus appropriée du thème du destin. Par la suite, les deux personnages ne cesseront de revenir sur l'événement et sur ce qu'il signifie pour leur histoire personnelle, selon les aléas de leur relation amoureuse. De la sorte, la musique est devenue un matériau romanesque qui traverse d'un bout à l'autre la partition du roman. Mais à l'inverse, dans le même roman où elle est comme le reflet inversé de Teresa, Sabina se méfie de la musique et s'oppose ainsi à Frantz, son amant. À celui-ci qui lui demande si elle aime la musique, elle répond :

- Non [...]. Puis elle ajoute : "peut-être que si je vivais à une autre époque..." et elle pense à l'époque de Jean-Sébastien Bach où la musique ressemblait à une rose épanouie sur l'immense plaine neigeuse du silence.

Le bruit sous le masque de la musique la poursuit partout. (p. 138)

Suit alors le souvenir des chantiers de jeunesse où il n'était possible nulle part d'échapper à la musique, artificiellement gaie de surcroît. Et Sabina de songer : "La Musique était comme une meute de chiens lâchés sur elle.» (Ibid.) Comment ne pas être frappé ici par le contrepoint d'une comparaison filée très lyriquement nostalgique et de la métaphore antilyrique qui vient la contredire? Mais Frantz ne saurait comprendre sa maîtresse, lui qui perçoit dans la musique «l'art qui se rapproche le plus de la beauté dionysiaque conçue comme ivresse» (p. 137). Nous ne sommes pas très loin ici de l'extase déjà analysée, et il s'agit là évidemment d'un contre modèle négatif et romantique, qui doit être versé au compte de la bêtise et du kitsch. J'en citerai un dernier avatar dans L'Immortalité. Alors que Laura la musicienne en tient pour la grande musique kitschifiée, sa nièce Brigitte ne jure que par le rock. Or, bien qu'elles croient s'opposer sur leurs goûts musicaux, la tante et la nièce ne font qu'enfler symétriquement leur ego au nom des mêmes droits réputés imprescriptibles du sentiment ${ }^{46}$. La leçon $s^{\prime}$ impose d'elle-même et les discrédite au même titre, car ce qui les divise est bien moins profond que ce qui les unit : non bien sûr le fait d'aimer la musique ou telle musique, 
mais l'affirmation de soi à travers celle de la musique établie en critère absolu du sentiment. C'est pourquoi, à l'inverse et pour les mêmes raisons, les personnages chers à Kundera entretiennent une relation de défiance, voire de rejet à l'égard de la musique : Tamina dans Le Livre du rire et de l'oubli, Sabina dans L'Insoutenable Légèreté de l'être ou Agnès dans L'Immortalité. Pour échapper à la bêtise, la musique ne peut valoir que comme ce qui appartient déjà toujours au passé, et se révèle irrémédiablement perdu.

Contre la bêtise de la musique, Milan Kundera en tient donc pour une conception exigeante et presque héroïque, mais, comme on a pu le voir, nullement exclusive d'un héritage populaire authentique : son élitisme d'artiste n'a rien de social. Ainsi, sa conception de l'art oppose à la dictature du sentiment une raison sensible qui tente de concilier intelligence et beauté ${ }^{47}$; tandis que l'attitude kitsch, sous le même nom d'art, ne promeut à son insu que l'image grimaçante du grotesque. Cette image, le romancier la ressaisit à son tour et s'emploie à la retourner contre la bêtise. Car parler de la musique pour Kundera, c'est toujours affirmer les droits et la suprématie de la littérature, et par-dessus tout sa propre vocation de romancier. Outre le refus de tout lyrisme (identifié à la poésie), je me risquerai à ajouter que c’est la nostalgie de la musique (non lyrique) qui a fait de Kundera un romancier. Ainsi, bien qu'appartenant à cette ère de la fin que nous avons l'impression de vivre, le roman serait le seul art à pouvoir progresser "contre le progrès du monde ${ }^{48}$ ». Pourtant, cette affirmation volontariste est, me semble-t-il, au moins partiellement remise en cause par la conscience mélancolique de l'écrivain, qui ne peut mesurer l'Histoire du roman qu'à l'aune de sa propre inscription dans cette Histoire : comme si, d'une certaine façon, il se percevait lui-même comme le dernier romancier. Il y a là pour finir une nostalgie qui nourrit d'un bout à l'autre de l'œuvre un tout autre lyrisme, sans ambition de victoire, et dont l'ironie n'est que l'autre face : quelques exemples ont pu en donner un aperçu, ne serait-ce que sur le plan du style, et contribuer ainsi à sa réhabilitation au sein même de l'œuvre de Kundera. Car s'il faut l'en croire, la bêtise de la musique et sa mort annoncée n'ouvrent que deux possibilités existentielles et esthétiques : la mélancolie et le rire. Par réaction, la «haine» qui en découle relève d'un regard certes lucide et désenchanté sur l'homme, mais en cela même profondément humain, et qui plus est humaniste : témoignant d'une vision et affirmant des valeurs. Il n'est pas d'autre parti pour un artiste et un romancier.

47. Dans La Lenteur, Gallimard, coll. «NRF», I995, p. 4I, M. Kundera évoque une «raison douce et tendre» : raison qu'en ce sens on peut qualifier d' «heureuse».

48. L'Art du roman, op. cit., p. 35. 
